\title{
A Nonlinear Adaptive Filter for Gyro Thermal Bias Error Cancellation
}

\author{
Joseph M. Galante*† \\ NASA Goddard Spaceflight Center, Greenbelt, MD, 20771, USA \\ Robert M. Sanner ${ }^{\ddagger}$ \\ University of Maryland, College Park, MD, 20742, USA
}

\begin{abstract}
Deterministic errors in angular rate gyros, such as thermal biases, can have a significant impact on spacecraft attitude knowledge. In particular, thermal biases are often the dominant error source in MEMS gyros after calibration. Filters, such as MEKFs, are commonly used to mitigate the impact of gyro errors and gyro noise on spacecraft closed loop pointing accuracy, but often have difficulty in rapidly changing thermal environments and can be computationally expensive. In this report an existing nonlinear adaptive filter is used as the basis for a new nonlinear adaptive filter designed to estimate and cancel thermal bias effects. A description of the filter is presented along with an implementation suitable for discrete-time applications. A simulation analysis demonstrates the performance of the filter in the presence of noisy measurements and provides a comparison with existing techniques.
\end{abstract}

\section{Nomenclature}

$\begin{array}{ll}\boldsymbol{w}_{\text {meas }} & \text { angular rate measurement by a gyro } \\ \boldsymbol{w} & \text { true angular rate } \\ \boldsymbol{b}_{\text {const }} & \text { gyro bias constant (systematic error) } \\ \boldsymbol{b}_{\text {therm }} & \text { gryo thermal bias (systematic error) } \\ \boldsymbol{v} & \text { random gyro additive noise } \\ \hat{\boldsymbol{w}} & \text { angular rate estimate } \\ \hat{\boldsymbol{b}} & \text { bias estimate } \\ \boldsymbol{q} & \text { attitude quaternion } \\ \hat{\boldsymbol{q}} & \text { attitude estimate quaternion } \\ R(\boldsymbol{q}) & \text { rotation matrix parameterized by quaternion } \boldsymbol{q} \\ k & \text { scalar nonlinear adaptive observer tracking gain } \\ \alpha & \text { scalar nonlinear adaptive observer adaptation gain } \\ \boldsymbol{c} & \text { function approximation coefficient } \\ \Lambda & \text { unit triangle function } \\ t & \text { time } \\ T & \text { temperature }\end{array}$

\section{Introduction}

A fundamental problem for spacecraft control systems is the estimation of vehicle attitude and angular rate. Spacecraft often have stringent pointing requirements to accommodate science or mission payloads. Of course all physical attitude and angular rate sensors have stochastic noise and systematic errors in their measurements which can propagate through a spacecraft attitude controller and result in poor pointing

*Aerospace Engineer, Attitude Control Systems Engineering Branch, NASA Goddard Spaceflight Center.

$\dagger$ Graduate Student, University of Maryland College Park, and AIAA Student Member.

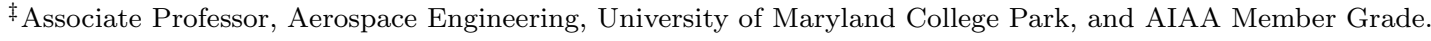


performance. The objective of an attitude filter is to decrease the impact of measurement noise and measurement error on the attitude control system. This is critically important for small spacecraft (such as CubeSats) since many small spacecraft do not have the mass or power budget to accommodate high quality sensors. Many small spacecraft use MEMS gyros as an angular rate measurement sensor that can meet these mass and power constraints, but MEMS gyros are well known to have large thermal bias errors that can change dramatically depending on the spacecraft's gyro's temperature.

In current spacecraft implementations, angular rate gyro systematic errors are typically modeled as constant biases and are estimated using a least squares approach or via an extended Kalman filter..$^{3-5,7}$ Vik et $a l^{12}$ proposed a new method that directly utilizes nonlinear quaternion kinematics but assumes biases that exponentially decay. Boskovic et $a l^{8,9}$ proposed a nonlinear bias observer based upon quaternion kinematics; however, the convergence proof assumes the vehicle attitude never passes through $\pm 180^{\circ}$. Thienel and Sanner ${ }^{1,2}$ proposed a nonlinear bias observer based upon the quaternion kinematics that they proved to be exponentially stable and singularity free; however the observer assumes a constant bias is the only systematic error in the gyro measurements. Mahony et al ${ }^{10,11}$ reformulated Thienel's and Sanner's observer in rotation matrix space and provided a variation of the observer design that reduces sensitivity to high frequency attitude noise at a cost of increased lag; but, similar to Thienel's and Sanner's work, the Mahony filter assumes a constant bias is the only systematic error in the gyro measurements.

None of the above filtering schemes directly address gyro thermal bias as a deterministic error source; instead all of the above approaches either model thermal bias as bias drift or lump in thermal bias with gyro noise. In this work, we consider the use of a collection of observers as in Thienel and Sanner, ${ }^{2}$ each set up to estimate bias at a specific (different) temperature. The bias estimates from all observers are then used in a function approximation scheme to estimate gyro bias as a function of temperature. This resulting bias estimate can thus be subtracted from the angular rate measurement to provide an estimate of the spacecraft angular rate even in the presence of rapid thermal variation of the gyro.

This paper is organized into several parts. Section II presents an angular rate gyro measurement model that includes a thermal bias. Next, Section III provides a brief overview of the nonlinear adaptive observer originally presented by Thienel and Sanner. ${ }^{2}$ A collection of those observers is then assembled into a new observer designed to estimate thermal bias in Section IV. A series of numerical simulations provides a comparison of the new observer with the observer developed by Thienel and Sanner as well as a popular extended Kalman filter in Section V. Finally, Section VI provides some key observations made by the authors and lists some future directions for the work.

\section{Measurement and System Models}

A gyro measurement model is shown in Eq. (1). In this model, $\boldsymbol{w}_{\text {meas }}(t)$ is the output of a gyro and is the only quantity directly available to an onboard flight computer. The goal of a gyro is to measure the true angular velocity of the spacecraft $\boldsymbol{w}(t)$, but the measurement is corrupted by constant bias $\boldsymbol{b}_{\text {const }}$, thermal bias $\boldsymbol{b}_{\text {therm }}(T(t))$, and random noise $\boldsymbol{v}(t)$. The constant bias $\boldsymbol{b}_{\text {const }}$ for a particular gyro can be determined via calibration on the ground and merely subtracted from gyro measurements; however, as no calibration is perfectly accurate and constant biases are known to slowly drift, it is common to refine an estimate of the bias in an attitude filter. Thermal bias, $\boldsymbol{b}_{\text {therm }}(T(t))$, is an additive bias that is an explicit function of gyro temperature $T(t)$ as depicted in Figure 1. This error source is modeled as a deterministic error, but as it is difficult to determine a calibration for this function some filters lump this error in with the random noise term or consider this error to be the source of bias drift. Finally, the random noise term $\boldsymbol{v}(t)$ is modeled as zero mean analog white Gaussian noise.

$$
\boldsymbol{w}_{\text {meas }}(t)=\boldsymbol{w}(t)+\boldsymbol{b}_{\text {const }}+\boldsymbol{b}_{\text {therm }}(T(t))+\boldsymbol{v}(t)
$$

The attitude of a spacecraft can be parameterized by a unit quaternion. A unit quaternion parameterizing attitude consists of a vector part $\epsilon$ and a scalar part $\eta$ as shown in Eq. 2. Eq. 2 also shows the relation between the unit quaternion parameterization of attitude and the Euler axis angle representation which consists of the Euler axis $\boldsymbol{e}$, a unit vector, and the Euler angle $\phi$.

$$
\boldsymbol{q}=\left[\begin{array}{l}
\boldsymbol{\epsilon} \\
\eta
\end{array}\right]=\left[\begin{array}{c}
\boldsymbol{e} \sin \frac{\phi}{2} \\
\cos \frac{\phi}{2}
\end{array}\right]
$$




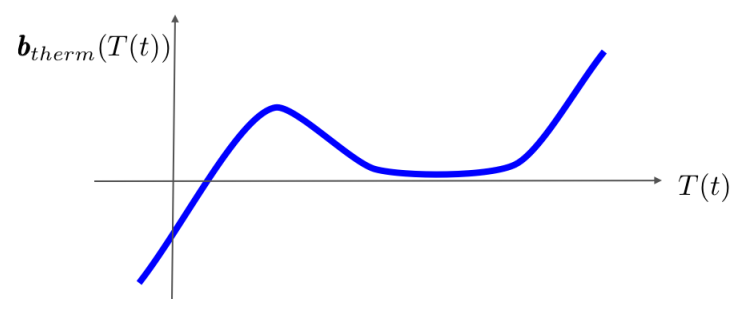

Figure 1. A complicated model of a thermal bias.

We let the unit quaternion represent the rotation from an inertial coordinate system to the spacecraft body coordinate system. Note that $\|\boldsymbol{q}\|=1$ by definition. The attitude quaternion can be used to construct a rotation matrix $R(\boldsymbol{q})=\left(\eta^{2}-\boldsymbol{\epsilon}^{T} \boldsymbol{\epsilon}\right) I+2 \boldsymbol{\epsilon} \boldsymbol{\epsilon}^{T}-2 \eta S(\boldsymbol{\epsilon})$ where $I$ is an identity matrix and $S(\boldsymbol{\epsilon})$ is a matrix representation of the cross product operation

$$
S(\epsilon)=\left[\begin{array}{ccc}
0 & -\epsilon_{z} & \epsilon_{y} \\
\epsilon_{z} & 0 & -\epsilon_{x} \\
-\epsilon_{y} & \epsilon_{x} & 0
\end{array}\right]
$$

The relative orientation between coordinate frames represented by $\boldsymbol{q}_{1}$ and $\boldsymbol{q}_{2}$ can be computed via

$$
\tilde{\boldsymbol{q}}=\left[\begin{array}{c}
\tilde{\boldsymbol{\epsilon}} \\
\tilde{\eta}
\end{array}\right]=\boldsymbol{q}_{1} \otimes \boldsymbol{q}_{2}^{-1}=\left[\begin{array}{cc}
\eta_{2} I-S\left(\boldsymbol{\epsilon}_{2}\right) & \boldsymbol{\epsilon}_{2} \\
\boldsymbol{\epsilon}_{2}^{T} & \eta_{2}
\end{array}\right]\left[\begin{array}{c}
\boldsymbol{\epsilon}_{1} \\
\eta_{1}
\end{array}\right]
$$

where $\tilde{\boldsymbol{q}}$ defines the rotation from the frame defined by $\boldsymbol{q}_{2}$ to the frame defined by $\boldsymbol{q}_{1}$. Note that when $\|\tilde{\boldsymbol{\epsilon}}\|=0$ and $\tilde{\eta}= \pm 1$, frame 2 is aligned with frame 1 .

The attitude kinematics of the spacecraft can be described with the differential equation

$$
\dot{\boldsymbol{q}}=\frac{1}{2} Q(\boldsymbol{q}(t)) \boldsymbol{w}(t)
$$

where

$$
Q(\boldsymbol{q}(t))=\left[\begin{array}{c}
\eta(t) I+S(\boldsymbol{\epsilon}(t)) \\
-\boldsymbol{\epsilon}^{T}(t)
\end{array}\right]
$$

Finally, we use Eq. 4 to model attitude measurement. The attitude measurement $\boldsymbol{q}_{\text {meas }}(t)$ is simply the true attitude $\boldsymbol{q}(t)$ corrupted by a small noise rotation $\boldsymbol{q}_{\text {noise }}(t)$. The noise rotation is constructed using a small angle assumption, in which case the quaternion noise properties can be specified in terms of the $\mathrm{x}, \mathrm{y}$, and $\mathrm{z}$ axes of the spacecraft. This is useful for specifying, for example, the larger noise about bore sight than about transverse axes which is typical for a star tracker. The noise rotation is constructed according to Eq. 5 , where $\theta_{x}, \theta_{y}$, and $\theta_{z}$ are each chosen from zero mean normal distributions with variances chosen to model attitude sensor performance.

$$
\begin{gathered}
\boldsymbol{q}_{\text {meas }}(t)=\boldsymbol{q}(t) \otimes \boldsymbol{q}_{\text {noise }}(t) \\
\boldsymbol{q}_{\text {noise }}(t)=\boldsymbol{q}_{x}\left(\theta_{x}(t)\right) \otimes \boldsymbol{q}_{y}\left(\theta_{y}(t)\right) \otimes \boldsymbol{q}_{z}\left(\theta_{z}(t)\right)
\end{gathered}
$$

where

$$
\boldsymbol{q}_{x}\left(\theta_{x}(t)\right)=\left[\begin{array}{c}
\sin \left(\frac{\theta_{x}(t)}{2}\right) \\
0 \\
0 \\
\cos \left(\frac{\theta_{x}(t)}{2}\right)
\end{array}\right] \quad \boldsymbol{q}_{y}\left(\theta_{y}(t)\right)=\left[\begin{array}{c}
0 \\
\sin \left(\frac{\theta_{y}(t)}{2}\right) \\
0 \\
\cos \left(\frac{\theta_{y}(t)}{2}\right)
\end{array}\right] \quad \boldsymbol{q}_{z}\left(\theta_{z}(t)\right)=\left[\begin{array}{c}
0 \\
0 \\
\sin \left(\frac{\theta_{z}(t)}{2}\right) \\
\cos \left(\frac{\theta_{z}(t)}{2}\right)
\end{array}\right]
$$




\section{Constant Bias Observer}

A novel approach to the gyro filtering problem using nonlinear adaptive techniques was introduced by Thienel and Sanner. ${ }^{2}$ This filter, which will hereafter be referred to as a Constant Bias Observer (CBO), does not assume an explicit model for thermal bias as a function of temperature; instead it models the gyro bias as a constant with drift that is slow compared to the dynamics of the filter. A clear distinction between this filter and an extended Kalman filter is that the CBO assumes the star tracker measurements $\boldsymbol{q}_{\text {meas }, k}$ are perfect. The CBO treats any discrepancy between the star tracker measurements and the gyro measurements as the fault of the gyro. Any resulting discrepancy is then used as an error metric to both drive convergence of the filter for angular rate estimation and to drive an adaptive law to learn a constant gyro bias. A direct consequence of the design of this filter is that the attitude estimates produced by the $\mathrm{CBO}$ are in general worse estimates of system attitude than the raw star tracker measurements.

The CBO equations originally presented by Thienel and Sanner ${ }^{2}$ are repeated below for convenience. The $\mathrm{CBO}$ produces an estimate of the spacecraft angular rate by subtracting a bias estimate from the measured angular rate as in Eq. 6 . The CBO generates a bias estimate by comparing its quaternion estimate $\hat{\boldsymbol{q}}(t)$ with the measured attitude as in Eq. 7. The resulting quaternion difference $\tilde{\boldsymbol{q}}(t)$ is then used to form an error metric term, $\tilde{\boldsymbol{\epsilon}}(t) \operatorname{sign}(\tilde{\eta}(t))$, to drive the estimator dynamics. The CBO's quaternion estimate is driven according to Eq. 8. Note that Eq. 8 is a copy of the attitude kinematics, Eq. 3, with the error metric term added to the angular velocity estimate and an extra rotation $R^{T}(\tilde{\boldsymbol{q}}(t))$ to resolve the angular velocity terms in the observer frame. The scalar $k$ that multiplies the error metric acts as a gain that controls the rate of convergence of the observer. Indeed, when the attitude estimate $\hat{\boldsymbol{q}}$ matches the measured attitude $\boldsymbol{q}$, the error metric term goes to zero and the estimator kinematics equation Eq. 8 simplifies to $\dot{\hat{\boldsymbol{q}}}(t)=\frac{1}{2} Q(\hat{\boldsymbol{q}}(t)) \hat{\boldsymbol{w}}(t)$, effectively copying the true kinematics of Eq. 3. Finally, the bias estimate $\hat{\boldsymbol{b}}(t)$ evolves according to the adaptive law given in Eq. 9 which consists of multiplying the error metric by a scalar adaptive gain $\alpha$ that controls the rate of adaptation of the bias estimate.

$$
\begin{gathered}
\hat{\boldsymbol{w}}(t)=\boldsymbol{w}_{\text {meas }}(t)-\hat{\boldsymbol{b}}(t) \\
\tilde{\boldsymbol{q}}(t)=\left[\begin{array}{c}
\tilde{\boldsymbol{\epsilon}}(t) \\
\tilde{\eta}(t)
\end{array}\right]=\boldsymbol{q}_{\text {meas }}(t) \otimes \hat{\boldsymbol{q}}^{-1}(t) \\
\dot{\hat{\boldsymbol{q}}}(t)=\frac{1}{2} Q(\hat{\boldsymbol{q}}(t)) R^{T}(\tilde{\boldsymbol{q}}(t))[\hat{\boldsymbol{w}}(t)+k \tilde{\boldsymbol{\epsilon}}(t) \operatorname{sign}(\tilde{\eta}(t))] \\
\dot{\hat{\boldsymbol{b}}}(t)=-\frac{1}{2} \alpha \tilde{\boldsymbol{\epsilon}}(t) \operatorname{sign}(\tilde{\eta}(t))
\end{gathered}
$$

The CBO has a number of distinctive properties. For the case of no random noise, both the CBO's angular rate estimate $\hat{\boldsymbol{w}}(t)$ and gyro bias estimate $\hat{\boldsymbol{b}}(t)$ are guaranteed to converge to their true values exponentially fast. For the case of random gyro noise, the $\mathrm{CBO}$ is provably stable. This filter requires no linearization. Thienel and Sanner proved that the CBO, when coupled with a stable attitude controller, exhibits the separation principle; that is, the $\mathrm{CBO}$ is guaranteed to result in a stable closed loop system. Again, note that the CBO models only a constant gyro bias. If the gyro has a time-varying bias and the gyro temperature changes sufficiently fast, the CBO's bias estimate $\hat{\boldsymbol{b}}(t)$ will lag the true bias.

For implementation in our simulation, or for an actual flight mission, the differential equations in the CBO need to be integrated. To integrate the quaternion estimate Eq. 8, we use the quaternion kinematic integration technique described on page 565 of Wertz. ${ }^{14}$ We use Euler quadrature to integrate Eq. 9.

\section{Thermal Bias Observer}

The Thermal Bias Observer, or TBO for short, is a new extension to the CBO discussed in the previous section. While the $\mathrm{CBO}$ is proven, given some assumptions, to be able to learn a constant bias, the CBO has a steady state error when trying to track a bias that varies with temperature. The steady state error of the gyro bias estimation is proportional to the rate of change of the gyro temperature. The concept behind the TBO is to get around this difficulty by partitioning the temperature range and providing a bias estimate for each partition, effectively providing a model-independent approximation to the thermal bias function. 
A simple implementation of the concept of temperature range partitioning is illustrated in Figure 2. In this figure, the width of each blue box is the size of a given temperature partition; the height of each box is the bias estimate associated with that temperature partition. The TBO is essentially a collection of CBOs, one $\mathrm{CBO}$ for each temperature partition. The TBO also includes some simple logic to generate a bias estimate for the temperature partition corresponding to the current temperature of the gyro and to ensure that the TBO only updates the bias estimate for the current temperature partition. An immediate consequence of the design of the TBO is that the TBO can only estimate the thermal bias function for temperatures that the gyro has experienced; this is a physical example of a persistency of excitation condition as discussed in Khaliil ${ }^{13}$ and should be expected of any filter that does not have an explicit model of the thermal bias function.

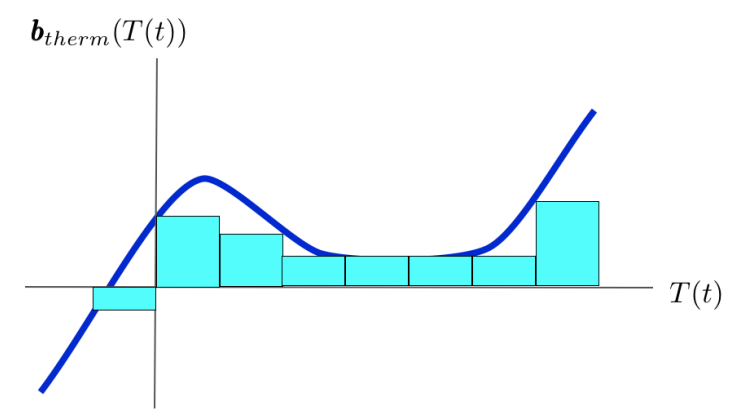

Figure 2. A simple implementation of the TBO partitions the temperature range and assigns a bias, represented as a blue box, for each partition. The TBO updates only the estimate for the partition which contains the current temperature. If the temperature variation repeatedly spans the entire temperature range, the TBO can estimate the entire thermal bias function.

Since the simple implementation of the TBO merely consists of a CBO for each temperature partition, the performance of the TBO will be identical to that of the CBO if the gyro temperature stays within a single temperature partition. Thus the TBO can be expected to have similar performance to the CBO for slowly varying thermal biases. The main improvement with this technique is that the bias estimate for each temperature partition is saved for future use. For example, consider the situation when the gyro dwells at a temperature in partition $A$ for some time, then abruptly changes to a temperature in partition $D$, dwells there for some time, then abruptly changes back to a temperature in partition $A$, and repeats. In this situation, the $\mathrm{CBO}$ would experience a transient in its bias estimate every time the temperature changed. The TBO in this situation, however, only experiences an initial transient during the first few temperature changes as the TBO's estimates of the thermal bias function in the temperature partitions converge. The TBO can be thought of as saving its work as it estimates the thermal bias. The TBO could also be said to approximate the thermal bias function with a set of rectangle basis functions where each temperature partition has a rectangle function with a width the size of the partition and unit height; multiplying a rectangle function by its corresponding bias estimate yields the blue rectangles depicted in Figure 2. Of course, this implementation of the TBO still experiences a steady state error in its bias estimate as the gyro temperature can vary within a temperature partition, but this error can be made arbitrarily small by dividing the temperature range into smaller partitions at a cost of increased initial settling time as there are more bias estimates.

If a thermal bias function can be modeled as a piece-wise continuous function, a slightly more sophisticated version of the TBO can be implemented. The idea behind the improvement is to construct an approximation of the thermal bias function by interpolating between bias estimates at the edges of each partition. This improvement can be realized by utilizing triangle basis functions such as in Eq. 10

$$
\Lambda\left(T_{\text {center }}, T_{\text {eval }}\right)= \begin{cases}1-\frac{\left|T_{\text {center }}-T_{\text {eval }}\right|}{T_{\text {width }}} & \text { for }\left|T_{\text {center }}-T_{\text {eval }}\right|<T_{\text {width }} \\ 0 & \text { otherwise }\end{cases}
$$

where $T_{\text {center }}$ is the location of the peak of the triangle function on the temperature axis, $T_{\text {eval }}$ is the temperature at which we evaluate the triangle function, and $T_{\text {width }}$ is the temperature partition width. 
Using many triangle functions, one centered at the end of each partition of the temperature range over which the observer is to estimate the gyro bias function, provides the basis functions needed to approximate the unknown gyro bias function as graphically illustrated in Figure 3. The triangle functions are each scaled by a coefficient and appear in the figure as thin green lines. Summing all the scaled triangle basis functions produces the thick green line which is a piece-wise continuous function approximation. This technique allows for a better function approximation and requires far fewer basis functions than the simple implementation with rectangle basis functions at only a very small computational cost increase; the TBO is still far computationally lighter than an extended Kalman filter.

The goal of the TBO is to thus estimate the scaling coefficients for each triangle basis function. This is almost equivalent to the operation of the CBO. If the gyro temperature never changes, then the TBO will update the scaling coefficients that correspond to the triangle functions that are nonzero at that temperature.

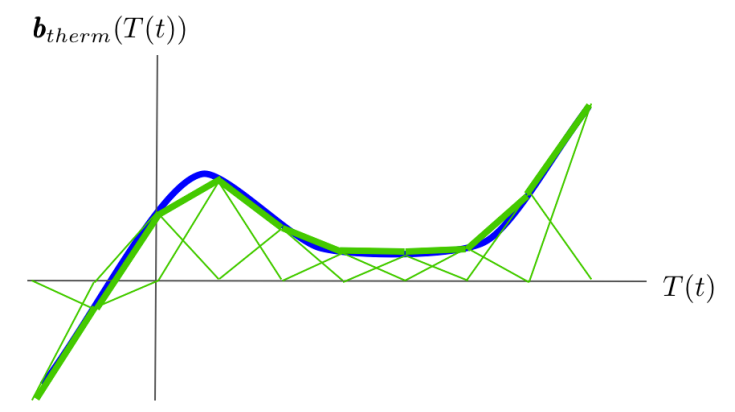

Figure 3. An implementation of the TBO using triangle basis functions to produce a piece-wise continuous approximation of the thermal bias function.

The TBO can be implemented as shown in Eqs. 11-17. Just as with the CBO, the TBO produces an estimate of angular rate by subtracting a bias estimate from the gyro measurement in Eq. 11. The TBO compares its attitude estimate with the attitude measurement in Eq. 12 which is subsequently used in an error metric $\tilde{\boldsymbol{\epsilon}}(t) \operatorname{sign}(\tilde{\eta}(t))$. The error metric is used in the TBO's differential equation for the quaternion estimate in Eq. 13 in the same way as in the CBO.

$$
\begin{gathered}
\hat{\boldsymbol{w}}(t)=\boldsymbol{w}_{\text {meas }}(t)-\hat{\boldsymbol{b}}(t) \\
\tilde{\boldsymbol{q}}(t)=\left[\begin{array}{c}
\tilde{\boldsymbol{\epsilon}}(t) \\
\tilde{\eta}(t)
\end{array}\right]=\boldsymbol{q}_{\text {meas }}(t) \otimes \hat{\boldsymbol{q}}(t)^{-1} \\
\dot{\hat{\boldsymbol{q}}}(t)=\frac{1}{2} Q(\hat{\boldsymbol{q}}(t)) R^{T}(\tilde{\boldsymbol{q}}(t))[\hat{\boldsymbol{w}}(t)+k \tilde{\boldsymbol{\epsilon}}(t) \operatorname{sign}(\tilde{\eta}(t))]
\end{gathered}
$$

Next, the TBO needs to update its bias estimate; however, instead of applying the bias adaptation law directly to the bias estimate as in the CBO, the TBO applies the bias adaptation law to the scaling coefficients $\hat{\boldsymbol{c}}(i, t)$ that are associated with the triangle basis functions. In general, we would need to apply the adaptive law to each coefficient in proportion to how much they contribute to the current bias estimate. If we had used a different choice of basis functions, such as radial basis functions, then we would need to update every triangle coefficient. With our choice of triangle basis functions, however, only two triangle functions are nonzero at any given temperature (or, equivalently, time). So the next step for the TBO is to determine which triangle basis functions are nonzero at the current temperature (time), which is accomplished with a simple search or lookup table. We note this step in Eq. 14; given the current temperature, the function findCurrentBin identifies the index of the temperature partition. We denote this temperature partition's index as bin.

$$
\text { bin }=\text { findCurrentBin }(T(t))
$$

The index bin is such that it also denotes the triangle basis function whose center is on the left end of the temperature partition. We can thus use the index bin to identify the coefficient for the triangle basis 
function whose center is on the left end of the temperature partition, $\hat{\boldsymbol{c}}($ bin, $t)$, and the coefficient for the triangle basis function whose center is on the right end of the temperature partition, $\hat{\boldsymbol{c}}($ bin $+1, t)$. This index allows us to apply the adaptive law $-\frac{\alpha}{2} \operatorname{sign}(\tilde{\eta}(t)) \tilde{\boldsymbol{\epsilon}}(t)$ to the appropriate triangle basis function scaling coefficients in proportion to their current contribution to the bias estimate as in Eq. 15-16. Finally, the new bias estimate is constructed by summing the value of the scaled triangle basis functions at the current temperature as in Eq. 17.

$$
\begin{gathered}
\dot{\hat{\boldsymbol{c}}}(\operatorname{bin}, t)=-\frac{\alpha}{2} \operatorname{sign}(\tilde{\eta}(t)) \tilde{\boldsymbol{\epsilon}}(t) \Lambda(\operatorname{locations}(\operatorname{bin}), T(t)) \\
\dot{\hat{\boldsymbol{c}}}(\operatorname{bin}+1, t)=-\frac{\alpha}{2} \operatorname{sign}(\tilde{\eta}(t)) \tilde{\boldsymbol{\epsilon}}(t) \Lambda(\operatorname{locations}(\operatorname{bin}+1), T(t)) \\
\hat{\boldsymbol{b}}(t)=\hat{\boldsymbol{c}}(\operatorname{bin}, t) \Lambda(\operatorname{locations}(\operatorname{bin}), T(t))+\hat{\boldsymbol{c}}(\operatorname{bin}+1, t) \Lambda(\operatorname{locations}(\operatorname{bin}+1), T(t))
\end{gathered}
$$

\section{Numerical Simulation Results}

This section presents a collection of preliminary simulation results comparing the performance of both of the above mentioned filtering algorithms. Additionally, a Multiplicative Extended Kalman Filter, or MEKF, as described by Markley ${ }^{6}$ is included in the simulation study for comparison purposes. All simulations are for a rigid nadir-pointing spacecraft at LEO. A thermal bias is assumed for all simulations, but is merely a linear function of temperature. A variety of sensor suites were considered; the noise parameters of those sensor suites are summarized in Table 1. Each filter was discretized and was provided measurements every $\Delta t_{\text {measupdate }}$ seconds.

\begin{tabular}{|c||c|c|c|c|}
\hline Noise Level & None & Low & Medium & High \\
\hline \hline Hardware Grade & Ideal & Navigation & Tactical & Commercial \\
\hline$\Delta t_{\text {meas update }}(\mathrm{s})$ & 0.2 & 0.2 & 0.5 & 0.5 \\
\hline$\sigma_{\boldsymbol{v}}\left({ }^{\circ} \mathrm{hr} \sqrt{\mathrm{Hz}}\right)$ & - & 0.0090 & 2.1 & 17.1 \\
\hline$\sigma_{\boldsymbol{q}_{\text {noise }}\left({ }^{\circ}\right)}$ & - & 0.0011 & 0.019 & 0.10 \\
\hline
\end{tabular}

Table 1. Noise parameters for the various sensor suites considered.

For each of the simulations detailed below, the filters were tuned to the sensor models. This is straightforward for the MEKF as all of its tuning parameters are direct sensor specifications with the exception of the estimate of the standard deviation of the bias drift rate. Since the thermal bias changes slowly for small thermal changes, the variation of the thermal bias was assumed to be the dominant source of bias drift and used to tune the MEKF. It is common to alter the tuning of an MEKF from its theoretically optimal tuning based on heuristics and past experience; however, for this study the theoretically optimal tuning was chosen.

While a majority of the tuning parameters for the MEKF can be optimally chosen using sensor noise specifications, the authors are unaware of any explicit optimization criteria for the nonlinear filter gains $k$ (tracking gain) or $\alpha$ (bias estimate adaptation gain) based on sensor models. Instead, a brute-force method was utilized to choose the gains for both the CBO and TBO. For each sensor suite listed in Table 1, 625 simulations were performed with various guesses for $k$ and $\alpha$. The performance of the nonlinear filters for each simulation, measured by each filter's root mean square (RMS) angular rate estimation error, was then used to construct a plot as seen in Figures 4 and 5.

There are several nuances to using this analysis as an ad hoc gain tuning paradigm. If the initial bias estimate for the nonlinear filters is chosen to be very accurate, the resulting gain performance plots will show poor performance for sets of gain values that favor adaptation since the filter starts with a good guess. Similarly, if the initial bias estimate is off by a substantial margin but the simulation duration is set to be very long, the resulting gain performance plots will favor steady state filter performance, again favoring sets of gain values that have weak adaptation. Conversely, setting the simulation duration to be a short period of time with a poor initial bias estimate yields gain performance plots that indicate the best performance for filters with aggressive adaptation. Furthermore, the simulation scenario was programmed to have temperature vary sinusoidally with a period of 20 minutes. 
For the purposes of this study, the gains were chosen by setting the initial bias estimate to be off by $10 \%$ and the simulation duration to 2 hours. After completing the simulation experiments detailed in this report, it was clear that these gains were not the optimal choice for the simulation studies that follow. It is possible to tailor the above gain tuning procedure to more accurately reflect the scenario for which a filter will be used and thus produce a choice of gains that lead to better performance. Unfortunately, this brute force tuning strategy can require a prohibitive amount of computing to try out many different gain choices for simulations of long duration.
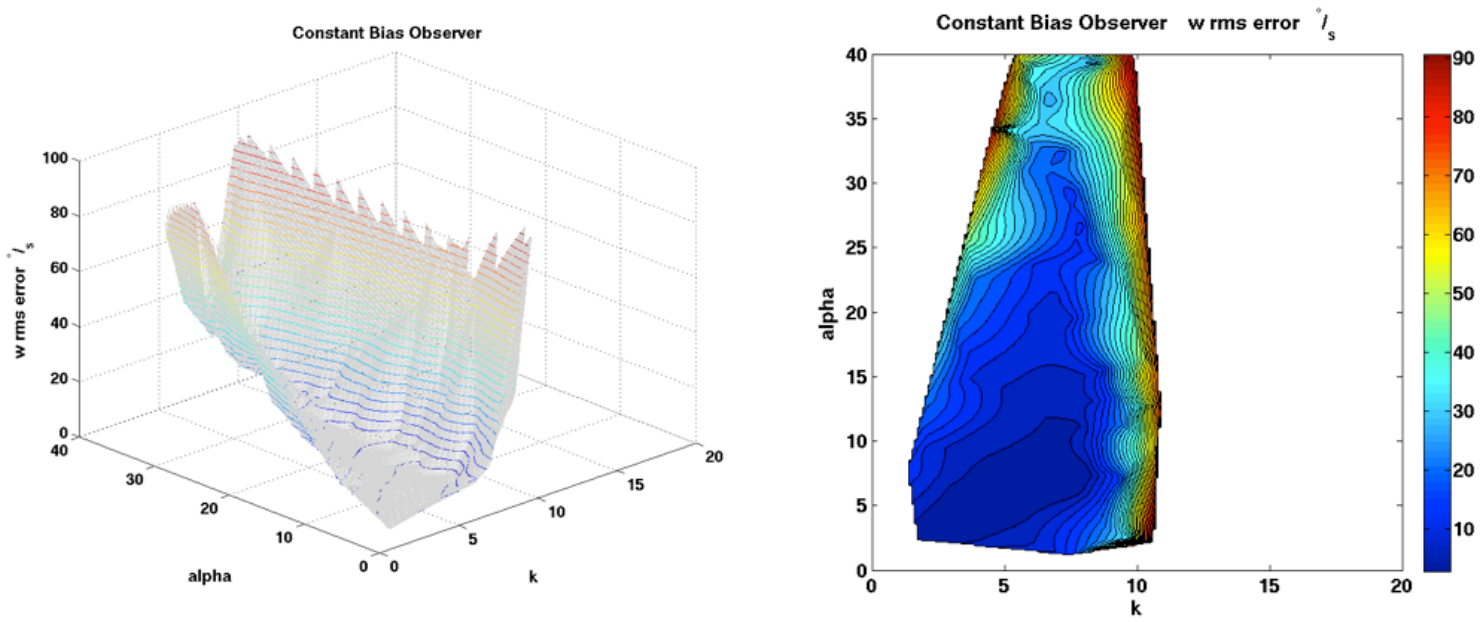

Figure 4. Two views of the measure of performance for various choices of gains for the CBO filter. In the left plot, the three dimensional shape of the performance can be easily visualized. The right plot, an overhead view of the same data, depicts good performance in blue and poor performance in red.
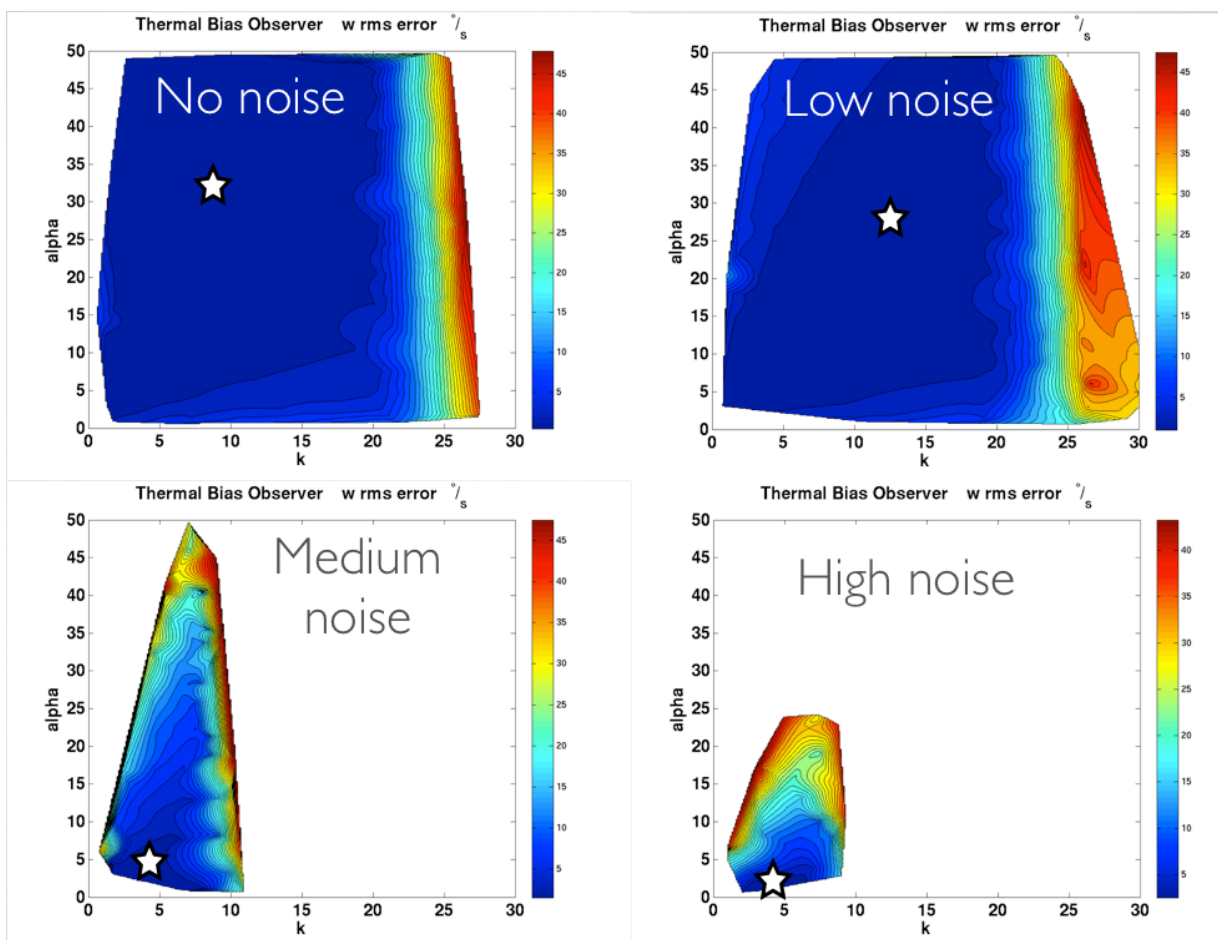

Figure 5. Overhead views of the TBO's performance for various noise conditions (detailed in Table 1). The filter gains chosen we chose are depicted with a star. Note that the filter's performance varies dramatically for different noise conditions. 
An analysis of the transient performance of the three filters was performed. For the transient analysis, all filters were loaded with an initial bias estimate that was off by $10 \%$. For the case of no noise, Figure 6 shows the transient response of the three filters across two timescales. The left plot indicates that all three filters experience a transient on the order of a few seconds. Over a longer timescale, as shown in the right plot in Figure 6, it is clear that the transient for the TBO actually occurs over a period of many hours. In this simulation the TBO did not reach steady state until approximately 35 hours into the simulation. The longer transient can be attributed to the TBO learning 15 coefficients for its approximation of the gyro thermal bias functions while the CBO and MEKF each only need to learn three bias estimates.
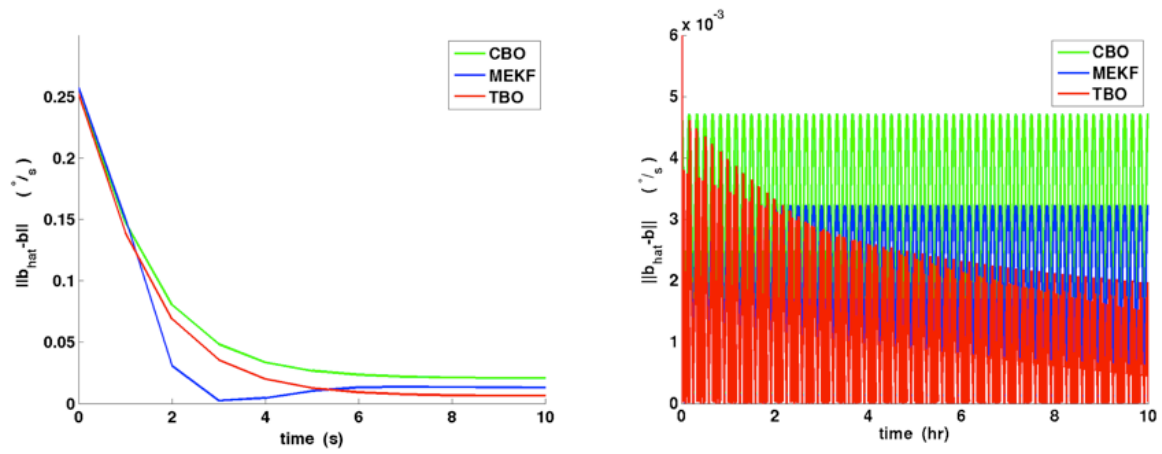

Figure 6. For the case of no random noise and an incorrect initial bias estimate, the transient response of the CBO and MEKF occur over a very short time scale as shown in the plot on the left. The right plot makes clear, however, that the TBO's transient is much longer.

Adding random measurement noise to the simulations dramatically reduces the settling time of the TBO as shown in Figure 7. It was surprising to see that all three filters performed similarly for all noise levels. The theoretical proof for the CBO makes no guarantees of filter performance in the presence of attitude noise but does state that the CBO is exponentially stable, so the performance of the CBO and the TBO in the presence of random attitude noise was of great interest. In Figure 7 the TBO not only has comparable performance to the MEKF in all noise scenarios, it also has a lower RMS error in all cases. Note carefully that the tuning of all three filters can be improved upon from the simulations seen here.

A study of the steady state performance of the three filters for various thermal periods was also performed. The above studies all model the temperature of the gyro as varying sinusoidally with a period of 20 minutes. In this analysis, 100 simulations were run, each simulation having a different thermal period. Figure 8 shows the results for the no noise (ideal sensor) case in axes-equal scale on the left plot and in a semilog scale in the right plot. As expected, all three filters perform similarly when thermal variation is very slow; the performance is similar on the right side of the performance plots. When the thermal variation is made faster, the thermal bias plays a more dominant role in the sensor error; the TBO continues to perform well while the $\mathrm{CBO}$ and MEKF incur more error due to the lag of their bias estimates trying to track the bias as the bias changes more rapidly.

The thermal period sensitivity analysis was repeated for the noisy sensor suites listed in Table 1 . The plots in Figure 9 indicate that the TBO performs better than the other filters for fast thermal variations as should be expected; the TBO is specifically designed to mitigate the effect of changing thermal bias. The TBO was able to perform the best even in the high noise scenarios for fast thermal variations. For medium and high noise scenarios, the MEKF was able to outperform the other filters only when the thermal variation was slow enough that the MEKF's bias estimate could sufficiently track the thermal bias. Note again that these results are contingent on the choice of gain and tuning parameters; it is likely that all three filters can be tuned to perform better in these scenarios. 

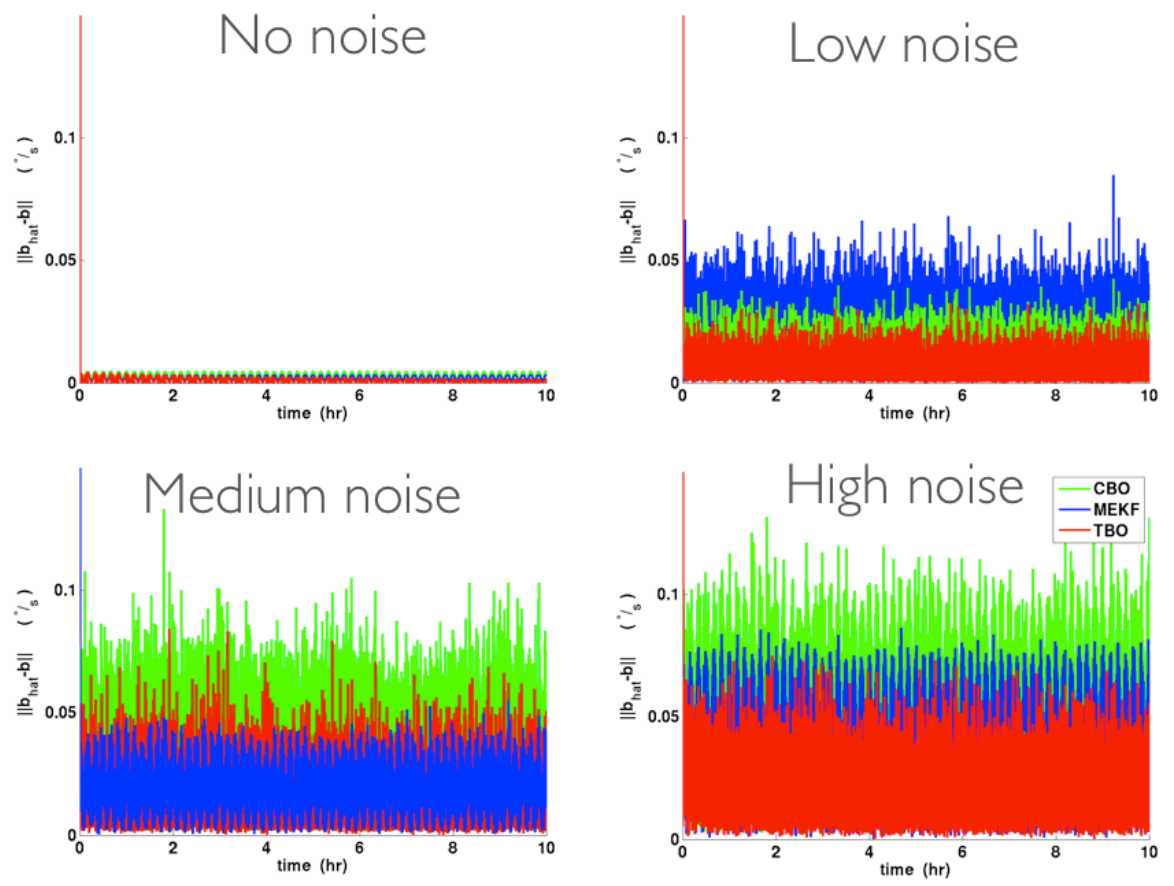

Figure 7. The transient response of all three filters is very fast when random noise is present. The RMS error of the TBO is smaller than that of the other filters for all cases even though the MEKF's error is more tightly bound for the medium noise case.
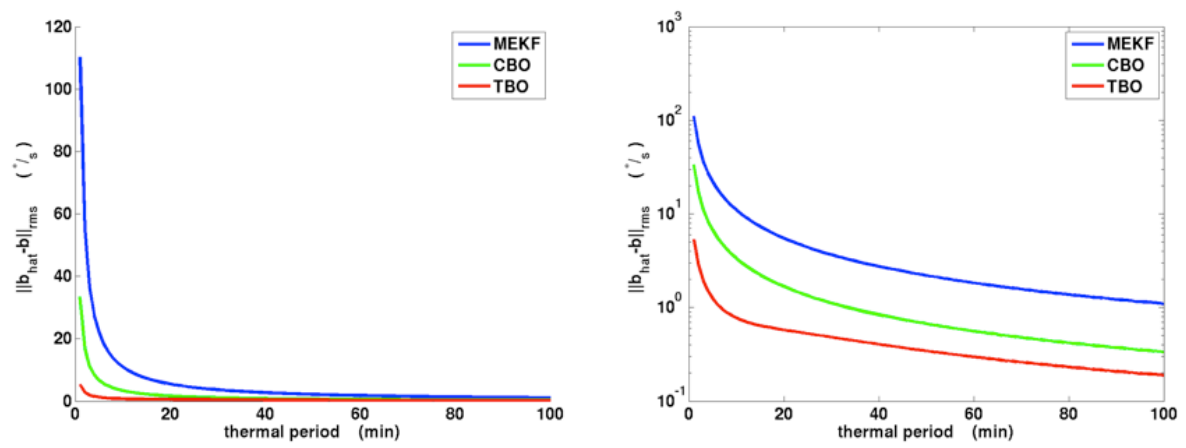

Figure 8. The period of the thermal variation is set to a different value for each of 100 simulations, producing an analysis of the sensitivity of each filter to the thermal period. The TBO performs substantially better than the other filters for fast thermal variation. As expected, the filters have similar performance for slow thermal variation as seen where the lines meet on the axes-equal plot (left). A semilog plot (right) reveals that the steady state error due to lagging bias estimates still result in a small but perceptible performance difference. 

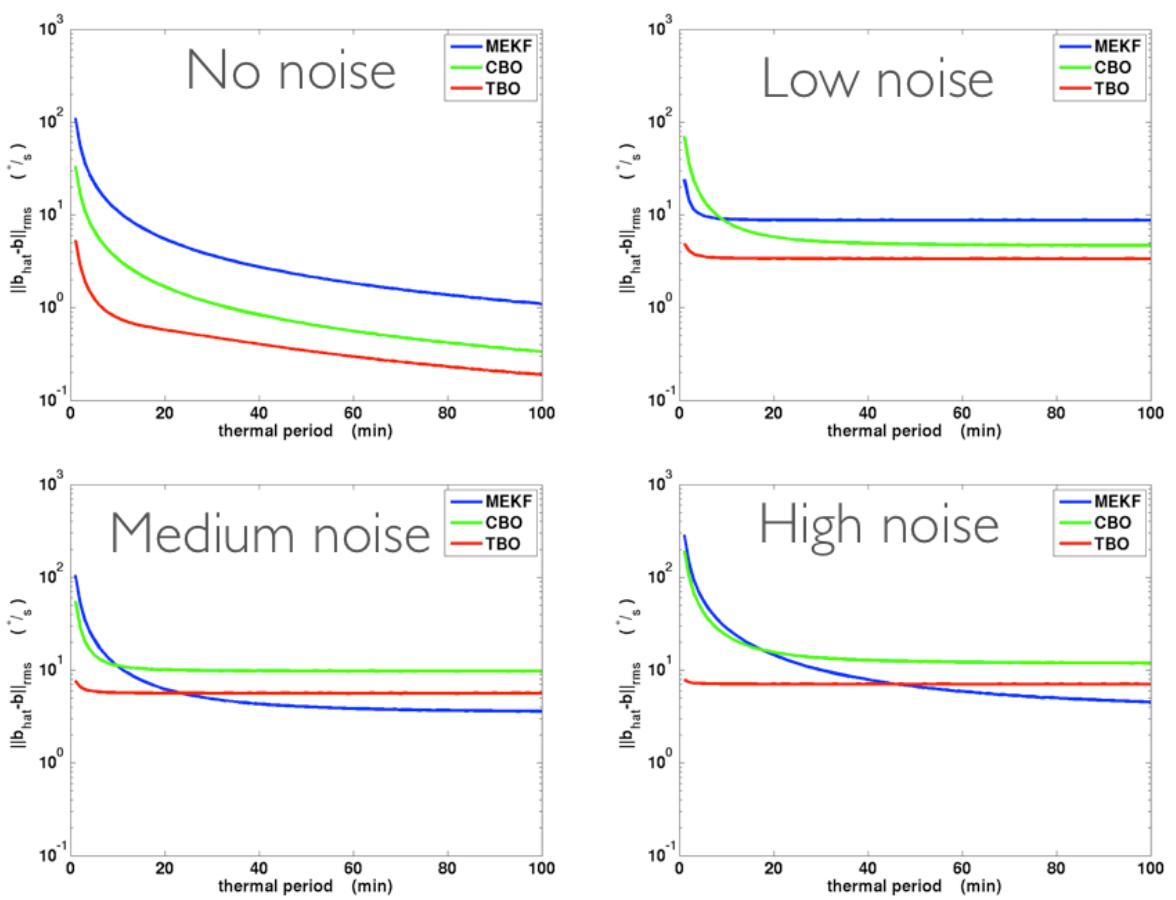

Figure 9. Repeating the thermal period sensitivity analysis for differing noise levels indicates the TBO performs the best for fast thermal variations. For high noise cases, the MEKF performs better so long as the thermal variation is slow enough that the MEKF's bias estimate can sufficiently track the thermal bias. 


\section{Conclusions and Future Work}

This report presented a nonlinear adaptive filter, the Thermal Bias Observer, for attitude rate estimation from gyro measurements subject to thermal bias errors. The TBO, comprised of a collection of CBOs and a function approximation technique, retains a number of the stability and identification properties of the CBO. A brute-force technique for tuning the nonlinear filter gains was presented. A simulation analysis studied the performance of the $\mathrm{CBO}$ and $\mathrm{TBO}$ nonlinear filters in the presence of measurement errors and random noise in comparison with an MEKF, a popular extended Kalman filter for attitude estimation. The MEKF and CBO performed well in all scenarios where the thermal variation was slow but had poor performance for fast thermal variation. The TBO performed well in all simulated scenarios. In particular, the TBO significantly outperformed the other filters when gyro thermal bias varied quickly. The TBO's excellent performance in situations with rapidly varying thermal biases along with its low computational complexity (compared to extended Kalman filter techniques) make the TBO a good candidate for future spacecraft missions with MEMS gyros.

This particular filter is of considerable interest to CubeSat missions with precise pointing requirements. Many CubeSats have low-cost MEMS gyros on board that are known for having significant bias variation due to temperature, a systematic error that this filter is designed to directly address. While this filter does require quaternion measurements, several star trackers designed for CubeSat missions are now available on the market. Additionally, quaternion measurements can be constructed from a pair of vector measurements using the TRIAD algorithm if a star tracker is not available; potential vector measurements include the local Sun vector from a fine or coarse sun sensor, the local Moon vector from a sun sensor, the local magnetic field using a magnetometer, the Earth horizon vector using a horizon sensor, or others.

There are several avenues for future development of this work. The nonlinear filters implemented in simulation were tuned with an ad hoc approach consisting of a brute-force search of the gain parameter space; the authors are presently working to derive a set of optimization criteria for the nonlinear adaptive filter gain parameters based on sensor specifications. The scope of simulation studies can easily be broadened; this study only considered simple thermal bias functions, only included slow spacecraft dynamics, and only permitted a certain class of thermal variations. All filters discussed here can be appropriately modified to treat gyro scale factor and alignment errors, common calibration issues that need to be resolved to make the filtering scheme directly applicable to flight projects. Additionally, the discretization approach implemented in the simulation software did not account for asynchronous measurements or sensor updates; these issues can be directly handled with more code but deserve a detailed study in their own right. Finally, the sensor models in the simulations performed were low fidelity, higher fidelity sensor models or even operation with hardware-in-the-loop would provide a more meaningful test of the filters' performance.

\section{Acknowledgments}

The authors would like to thank the Dextre Pointing Project and Argon GNC teams at NASA GSFC for their support in the development of this algorithm. In particular, John Van Eepoel was instrumental in securing resources for this work.

\section{References}

\footnotetext{
${ }^{1}$ Thienel, J.K., Nonlinear Observer/Controller Designs for Spacecraft Attitude Control Systems with Uncalibrated Gyros, Doctoral Thesis, 2004.

${ }^{2}$ Thienel, J.K., and Sanner, R.M. "A Coupled Nonlinear Spacecraft Attitude Controller and Observer with an Unknown Constant Gyro Bias and Gyro Noise," IEEE Trans. on Automatic Control, vol. 48, no. 11, pp. 2011-2015, Nov 2003.

${ }^{3}$ Hashmall, J.A., Radomski, M., and Sedlak, J. "On-orbit Calibration of Satellite Gyroscopes," presented at the AIAA/AAS Astrodynamics Specialist Conf., Denver, CO, Aug. 13-17, 2000.

${ }^{4}$ Welter, G., Boia, J., Gakenheimer, M., Kimmer, E., Channell, D., and Hallock, L. "Variations on the Davenport Gyroscope Calibration Algorithm," presented at the Flight Mechanics/Estimation Theory Symposium, Greenbelt, MD, May 14-16, 1996.

${ }^{5}$ Markley, F.L., and Reynolds, R.G. "Analytic Steady-State Accuracy of a Spacecraft Attitude Estimator," AIAA J. Guidance, Control, and Dynamics, vol. 23, no. 6, pp. 1065-1067, Nov.-Dec. 2000.

${ }^{6}$ Markley, F.L. "Attitude Error Representations for Kalman Filtering," AIAA J. Guidance, Control, and Dynamics, vol 26, no. 2, pp. 311-317, 2003.

${ }^{7}$ Farrenkopf, "Analytic Steady-State Accuracy Solutions for Two Common Spacecraft Attitude Estimators," AIAA J. Guidance, Control, and Dynamics, vol. 1, no. 4, pp. 282-284, July-Aug, 1978.
} 
${ }^{8}$ Boskovic, J.D., Li, S., and Mehra, R.K. "A Globally Stable Scheme for Spacecraft Control in the Presence of Sensor Bias," presented at the 2000 IEEE Aerospace Conference, Big Sky, MO, Mar 2000.

${ }^{9}$ Boskovic, J.D., Li, S., and Mehra, R.K. "Fault Tolerant Control of Spacecraft in the Presence of Sensor Bias," presented at the 2000 IEEE American Control Conference, Chicago, IL, June 2000.

${ }^{10}$ Mahony, R., Hamel, T., and Pflimlin, J.M. "Nonlinear Complementary Filters on the Special Orthogonal Group," IEEE Trans. on Automatic Control, vol. 53, no. 5, June 2008.

${ }^{11}$ Mahony, R., Hamel, T., and Pflimlin, J.M. "Complementary Filter Design on the Special Orthogonal Group SO(3)," presented at the 44th IEEE Conf on Decision and Control, Seville, Spain, Dec 12-15, 2005.

${ }^{12}$ Vik, B., Shiriaev, A., Fossen, T.I. "Nonlinear Observer Design for Integration of DGPS and INS," in New Directions in Nonlinear Observer Design, Lecture Notes in Control and Information Sciences. New York: Springer-Verlag, 1999 , pp. 135-159.

${ }^{13}$ Khalil, H. Nonlinear Systems. Upper Saddle River, NJ: Prentice-Hall, 2002.

${ }^{14}$ Wertz, J.R. Spacecraft Attitude Determination and Control. Dordrecht, Holland: D. Reidel Publishing Company, 1978. 